\title{
The isolated fossil group RX J1119.7+2126 (Research Note)
}

\author{
C. Adami ${ }^{1}$, D. Russeil ${ }^{1}$, and F. Durret ${ }^{2,3}$ \\ 1 LAM, Traverse du Siphon, 13012 Marseille, 2 place Le Verrier, 13248 Marseille, France \\ e-mail: christophe.adami@oamp.fr \\ 2 Institut d'Astrophysique de Paris, CNRS, UMR 7095, Université Pierre et Marie Curie, 98bis Bd Arago, 75014 Paris, France \\ 3 Observatoire de Paris, LERMA, 61 Av. de l'Observatoire, 75014 Paris, France
}

Received 1 June 2006 / Accepted 13 March 2007

\section{ABSTRACT}

\begin{abstract}
Context. Fossil groups are galaxy structures that probably underwent a nearly complete fusion of all intermediate magnitude galaxies into a single large central dominant galaxy. However, the formation and evolution processes of these structures are still not well understood.

Aims. In order to test this scenario and its implications we studied the fossil group RX J1119.7+2126, based on available spectroscopy of the galaxies in the low-density, large-scale region around the fossil group and deep B and R band imaging of its close vicinity and three comparison fields.

Methods. We used spectroscopic data to investigate the degree of isolation of RX J1119.7+2126 in terms of bright neighbor galaxies. The imaging data were used to derive the color-magnitude relation and select faint galaxies statistically belonging to this structure. Results. The structure appears as a very isolated group exhibiting a red sequence in the color magnitude relation with characteristics close to the red sequences already observed for other fossil groups.

Conclusions. All these results can be interpreted consistently in the framework of the building-up process generally proposed for fossil groups.
\end{abstract}

Key words. galaxies: clusters: individual: RX J1119.7+2126

\section{Introduction}

Hierarchical building of structures is a key ingredient for cosmological models, since galaxy structures such as groups and clusters are expected to be located at the intersections of cosmic filaments (e.g. Lanzoni et al. 2005, and references therein). These structures are then continuously fed by infalling field matter (galaxies and gas). However, a peculiar class of structures seems not to follow this general behaviour: fossil groups (e.g. Ulmer et al. 2005; Mendes de Oliveira et al. 2006, and references therein).

These structures are considered as the ultimate stage of group evolution: the nearly complete fusion of all the bright and intermediate magnitude galaxies of the group into a single bright galaxy. This resulting galaxy is brighter than the second remaining group galaxy (within half the projected virial radius) by at least 2 magnitudes (in the $R$ band). However, the extended X-ray gas envelope is still present and more luminous than $10^{42} h_{50}^{-2} \mathrm{erg} \mathrm{s}^{-1}$ (Jones et al. 2003). Recent numerical simulations by D'Onghia et al. (2005) have indeed shown that fossil groups have already assembled $50 \%$ of their final dark matter mass at $z \geq 1$ and that they subsequently grow by minor merging. D'Onghia et al. (2005) have also shown that they are expected to be overluminous in X-rays relatively to non-fossil groups. It was

* Based on observations made at Observatoire de Haute Provence (CNRS), France. Also based on the use of the NASA/IPAC Extragalactic Database (NED) which is operated by the Jet Propulsion Laboratory, California Institute of Technology, under contract with the National Aeronautics and Space Administration. also suggested in this paper that fossil groups exist only because infall of $L \sim L_{*}$ galaxies happens along filaments with small impact parameters.

We can also explain this lack of bright galaxies if fossil groups are isolated from any surrounding large scale cosmic structure during a time typically longer than the galaxy crossing time in the considered group (e.g. Sarazin 1986). In order to test this hypothesis, we carried out a detailed study of the fossil group RX J1119.7+2126 (RX J1119 hereafter).

RX J1119 is one of the less massive known fossil groups (Jones et al. 2003). It is located at $\alpha=11^{\mathrm{h}} 19^{\mathrm{mn}} 43.7^{\mathrm{s}}, \delta=$ $21^{\circ} 26^{\prime} 50^{\prime \prime}$ (J2000.0) and at a redshift of 0.061. This low redshift value enables it to be studied with moderately sized telescopes and its coordinates are outside any major recent survey such as the SDSS or 2dF. It is located on the same line of sight but significantly beyond, the well-known filaments embedding the Coma cluster. Its field of view is therefore heavily polluted by foreground galaxies.

In Sect. 2, we present our data and the characteristics deduced for this structure in terms of bright neighboring galaxies. Section 3 concerns the study of the faint galaxy population of RX J1119. Section 4 is the conclusion. In this paper we assume $H_{0}=75 \mathrm{~km} \mathrm{~s}^{-1} \mathrm{Mpc}^{-1}, \Omega_{\mathrm{m}}=0.3$ and $\Omega_{\Lambda}=0.7$. All magnitudes are given in the Vega system.

\section{Bright neighboring galaxies?}

The first goal of this paper is to investigate the degree of isolation of RX J1119 and the surrounding Large Scale Structure 
(LSS hereafter). The degree of isolation can be understood in terms of bright galaxies. In the model of a nearly complete fusion of the intermediate magnitude galaxies into a single bright galaxy, this galaxy is expected to be the dominant one of the neighboring space. The minimal size of the space to be considered is imposed by the fact that RX J1119 has to be isolated and therefore probably located in a void. The average size of the known voids is close to $40 \mathrm{Mpc}$ in diameter (Hoyle \& Vogeley 2004). We therefore tried to detect if there are brighter galaxies than the central RX J1119 galaxy in similar areas around the fossil group. In other words, is the RX J1119 central galaxy the brightest of its bubble (if located in such a bubble)?

In order to estimate (in a $7 \times 7 \mathrm{deg}^{2}$ area or $\sim 28 \times 28 \mathrm{Mpc}^{2}$, see below) the expected number of galaxies at $z \sim 0.061$ brighter than the RX J1119 central galaxy $\left(M_{\mathrm{R}}=-22\right.$ in our cosmology: Jones et al. 2003) and twice as bright as the RX J1119 central galaxy $\left(M_{\mathrm{R}}=-22.75\right)$, we used the field luminosity functions of Ilbert et al. (2005) and the cluster luminosity functions of Popesso et al. (2005). On the one hand, without any rich galaxy structures in the bubble, the field luminosity functions predict about 50 galaxies per magnitude bin brighter than $M_{\mathrm{R}}=-22$ in our sampled volume and less than 5 galaxies per magnitude bin brighter than $M_{\mathrm{R}}=-22.75$, respectively. On the other hand, if one or several rich clusters are present in the area, these numbers increase significantly: several dozen galaxies brighter than $M_{\mathrm{R}}=-22.75$ would be expected per magnitude bin.

In order to compare observations with these predictions, we compiled (from NED) all the redshifts known within a $12 \times 12 \mathrm{deg}^{2}$ area $\left(\sim 47 \times 47 \mathrm{Mpc}^{2}\right)$ whatever their magnitude. We limited the searched redshift range to [0.051, 0.071]. This range corresponds to a physical size of $\sim 90 \mathrm{Mpc}$ and \pm 3 times the galaxy velocity dispersion of a massive cluster. In this range, there are 20 galaxies in a $7 \times 7 \mathrm{deg}^{2}$ area (the area in which RX J1119 seems isolated, i.e. $\sim 28 \times 28 \mathrm{Mpc}^{2}$ ) and 143 in the $12 \times 12 \mathrm{deg}^{2}$ area. The closest known cluster is Abell 1145, only included in the outer region. We show these positions in Fig. 1 along with the two searched areas. Now, restricting this list to galaxies brighter than the central RX J1119 galaxy (magnitudes taken from the NED database), in the $7 \times 7 \mathrm{deg}^{2}$ area, there is only one galaxy (as compared to 50 from the expected numbers even without any rich galaxy structure in the field). This galaxy (also brighter than $M_{\mathrm{R}}=-22.75$ ) is, moreover, quite distant from the fossil group (about $12 \mathrm{Mpc}$ or slightly more than $3 \mathrm{deg}$ ). The redshift catalog is however not complete down to $M_{\mathrm{R}}=-22$ and we cannot exclude that a significant number of galaxies with unknown redshift maybe within the considered redshift range.

We then measured additional redshifts for 20 of the 23 galaxies extracted from NED (without the redshift information) in the largest area down to $M_{\mathrm{R}}=-22.75$ (plus one fainter galaxy that we caught at the same time as a brighter target). This produced a spectroscopic catalog $\sim 100 \%$ complete down to this magnitude. These spectroscopic observations were made at the Observatoire de Haute Provence with the $193 \mathrm{~cm}$ telescope and the Carelec spectrograph. We used the $133 \AA / \mathrm{mm}$ configuration (resolution $R=900$ ) and the useful spectral range was 3700-6700 $\AA$. This instrument is a single-slit device well adapted to such a survey: the target density is 0.16 per $\mathrm{deg}^{2}$ making high multiplex multiobject spectrographs inefficient. Results are given in Table 1.

None of these additional galaxies is in the [0.051, 0.071] redshift range: most are located around $z=0.023$, and 4 are around $z=0.035$. Therefore, there is only one galaxy with $M_{\mathrm{R}} \leq-22.75$ galaxies at the RX J1119 redshift and in the $7 \times 7 \mathrm{deg}^{2}$ area (assuming that the three galaxies with unknown

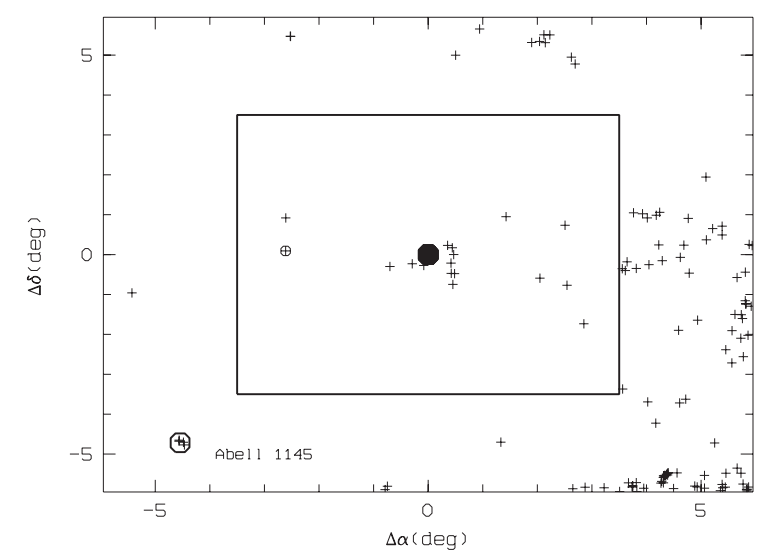

Fig. 1. Map of the $12 \times 12 \mathrm{deg}^{2}$ area around RX J1119 given in positions relative to the center of RX J1119. The large rectangle is the inner $7 \times$ $7 \mathrm{deg}^{2}$ area. Crosses are the galaxies with a redshift in the [0.051, 0.071] redshift range. Inside the $7 \times 7 \mathrm{deg}^{2}$ area, the only galaxy brighter than the RX J1119 central galaxy by more than 0.2 magnitude is shown as the small circled cross. The position of Abell 1145 is given at the bottom left of the plot. RX J1119 is shown by the central filled circle.

Table 1. Column 1: NED galaxy name; Col. 2: measured redshift; Col. 3: nature of the spectrum: emission lines (Em), absorption lines (Abs). The typical redshift uncertainty for these galaxies is 0.001 .

\begin{tabular}{lll}
\hline \hline Name & $z$ & Spectrum \\
\hline CGCG 125-023 & 0.023 & Em + Abs: Em dominated \\
UGC 06172 & 0.021 & Em + Abs: Em dominated \\
CGCG 096-008 & 0.026 & Abs only \\
CGCG 096-009 & 0.027 & Abs only \\
MCG +03-29-009 & 0.027 & Abs only \\
KUG 1110+235A & 0.023 & Em + Abs: Em dominated \\
KUG 1110+235 & 0.026 & Em + Abs: Em dominated \\
KUG 1112+236A & 0.022 & Em + Abs: Em dominated \\
CGCG 096-016 & 0.023 & Em + Abs: Abs dominated \\
CGCG 126-014 & 0.023 & Em + Abs: Em dominated \\
CGCG 126-018 & 0.023 & Abs only \\
UGC 06301 & 0.035 & Em + Abs: Em dominated \\
KUG 1115+236 & 0.022 & Abs only \\
CGCG 126-026 & 0.027 & Em + Abs \\
UGC 06336 & 0.026 & Abs only \\
CGCG 126-047 & 0.023 & Em + Abs: Abs dominated \\
CGCG 126-052 & 0.041 & Em + Abs: Em dominated \\
CGCG 126-055 & 0.024 & Em + Abs: Abs dominated \\
CGCG 126-076 & 0.032 & Em + Abs: Em dominated \\
KUG 1130+249A & 0.024 & Em + Abs: Em dominated \\
CGCG 126-088 & 0.034 & Abs only \\
\hline
\end{tabular}

redshift are not at $z=0.061$ ). This is clearly lower than the several dozen of galaxies expected if rich galaxy structures were present. This is even lower than the expected galaxy number assuming a "normal" field galaxy population.

RX J1119 therefore seems to be very isolated in terms of bright galaxies and it appears to be the dominant structure of its bubble. This bubble would have a typical diameter of $28 \mathrm{Mpc}$, close to the known void dimensions (Hoyle \& Vogeley 2004). However, this remains to be confirmed for faint galaxies with a redshift catalog complete down to fainter magnitudes than the one we have. For example, only less than $10 \%$ of all galaxies known in the $7 \times 7 \mathrm{deg}^{2}$ area (from NED) have a measured redshift. 


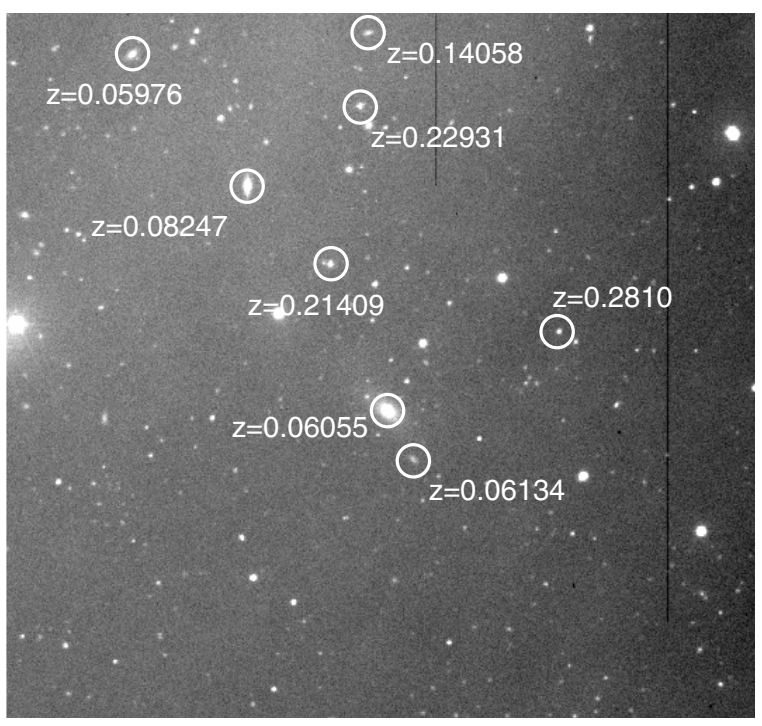

Fig. 2. $R$ band $10.2 \times 9.7 \operatorname{arcmin}^{2}$ image of RX J1119 with the known redshifts in the field.

Table 2. Column 1: observed field; Col. 2: coordinates (2000); Col. 3 : $R$ exposure time; Col. 4: $B$ exposure time; Col. 5: $E(B-V)$ following Schlegel et al. (1998).

\begin{tabular}{lllll}
\hline \hline Field & Coordinates (2000) & $R$ & $B$ & $E(B-V)$ \\
\hline RX J1119 & $111943.7,212650$ & $4500 \mathrm{~s}$ & $10800 \mathrm{~s}$ & $0.021 \pm 0.001$ \\
C1 & $234041.6,150711$ & $4500 \mathrm{~s}$ & $10800 \mathrm{~s}$ & $0.026 \pm 0.001$ \\
C2 & $010802.1,125627$ & $3600 \mathrm{~s}$ & $9720 \mathrm{~s}$ & $0.019 \pm 0.002$ \\
C3 & $022549.3,020945$ & $3240 \mathrm{~s}$ & $8400 \mathrm{~s}$ & $0.026 \pm 0.001$ \\
\hline
\end{tabular}

\section{The RX J1119 faint galaxy population}

\subsection{Imaging data}

We investigate in this section what happens at smaller scales, as the second goal of this research is to study the faint galaxy population in RX J1119 itself. Little is known of the galaxy populations in fossil groups beyond the spectroscopic limit and the second brightest galaxy for most of these structures (see however Mendes de Oliveira et al. 2006). Rather than measuring redshifts of galaxies of this population, we chose to obtain deep $B$ and $R$ band imaging data in order to draw a color magnitude relation (CMR hereafter). This was done at the OHP $120 \mathrm{~cm}$ telescope. A CCD camera with a $\sim 11.5 \times 11.5 \operatorname{arcmin}^{2}$ field $\left(1024 \times 1024\right.$ pixels with a pixel size of $\left.0.69 \times 0.69 \operatorname{arcsec}^{2}\right)$ is mounted on this telescope. We used Johnson $B$ and $R$ filters and we observed RX J1119 itself as well as three comparison fields (see Table 2). The comparison fields ( $\mathrm{C} 1, \mathrm{C} 2$ and $\mathrm{C} 3$ ) were chosen in order to have a Galactic extinction similar to that of RX J1119 (from the Schlegel et al. 1998 maps) and not to include nearby known galaxy structures. Data were acquired under photometric conditions and seeing of the order of 2.5 arcsec. The final useful area for RX J1119 with both $B$ and $R$ data available was $\sim 10.2 \times 9.7 \mathrm{arcmin}^{2}$. The three comparison fields cover $336 \operatorname{arcmin}^{2}$ in total and are distributed over a region of $\sim 45 \times 45 \mathrm{deg}^{2}$ on the sky. The images were calibrated using Landolt (1992) standard stars. We extracted catalogs of objects from these images using the SExtractor package (Bertin \& Arnouts 1996). An $R$ image of the RX J1119 field is displayed in Fig. 2 and the magnitude histograms are given in Fig. 3.

It is now important to perform a star-galaxy separation in order to avoid star pollution and to take into account the fact
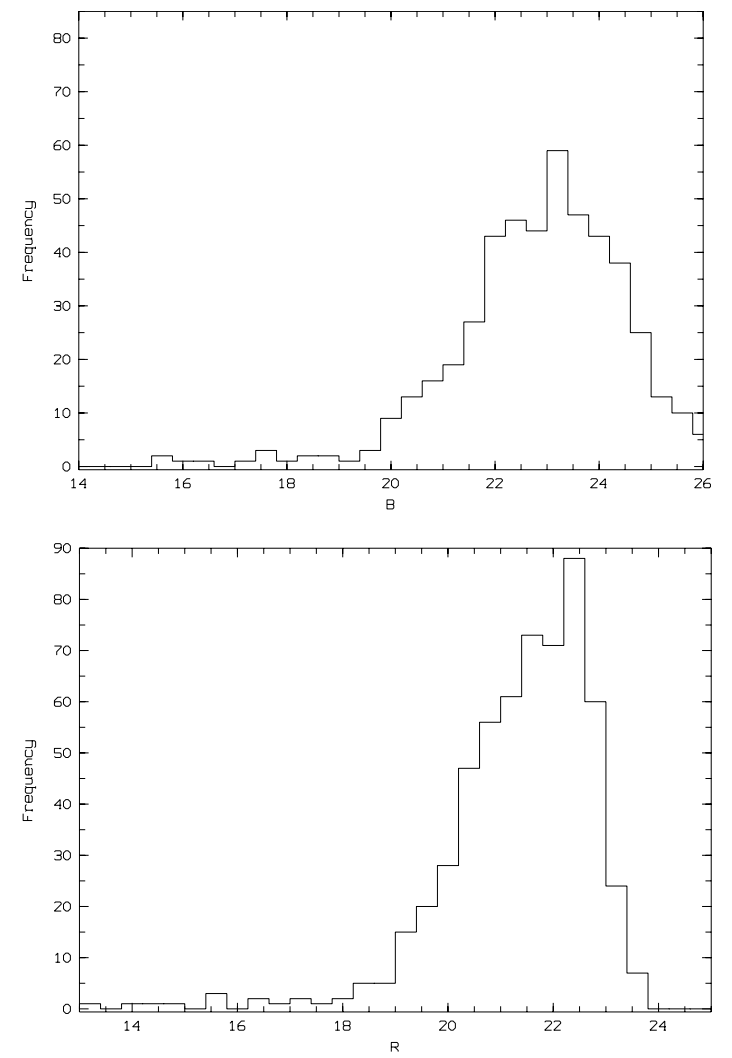

Fig. 3. Magnitude histograms of galaxies along the RX J1119 line of sight. Top: $B$ band, bottom: $R$ band.

that the four observed fields do not have the same Galactic latitude. Seeing conditions are not good enough to use the classical SExtractor star-galaxy separation flag. Instead, we used a total magnitude vs. central surface brightness plot (e.g. Adami et al. 2006) based on the deepest band data (the $R$ band). Results are illustrated in Fig. 4 for RX J1119, where we clearly see the star sequence. We were therefore able to make an efficient stargalaxy separation down to $R=20$ for the four observed fields. For fainter magnitudes, the surface-brightness profile of all objects is dominated by seeing effects and any star-galaxy separation would be very uncertain. However, the number of stars fainter than $R=20$ is small (Gazelle et al. 1995) and we decided to assume that all objects fainter than this limit are galaxies.

In order to test the separation down to $R=20$ for RX J1119, we retrieved in NED the 8 galaxies in the imaging field with a known redshift. We see that they are all classified as galaxies by our method in Fig. 4, including a relatively compact object.

\subsection{The $R X J 1119$ color magnitude relation}

We compute here the galaxy distribution in the $R / B-R$ plane for the RX J1119 structure. In order to achieve this goal, we have to subtract the fore and background contributions along the line of sight to RX J1119. We used a technique similar to that described in Adami et al. (2007). Briefly, the fore and background RX J1119 line of sight galaxy populations were estimated using the three comparison fields. For a given $R$ and $B-R$, the number of galaxies per $\operatorname{deg}^{2}$ in the RX J1119 structure $\left(N_{\mathrm{RX}} \mathrm{J1119}\right)$ was computed with the number of galaxies per $\mathrm{deg}^{2}$ along the RX J1119 line of sight ( $N_{\mathrm{RX}}$ J1119 l.o.s $)$ and the numbers of 


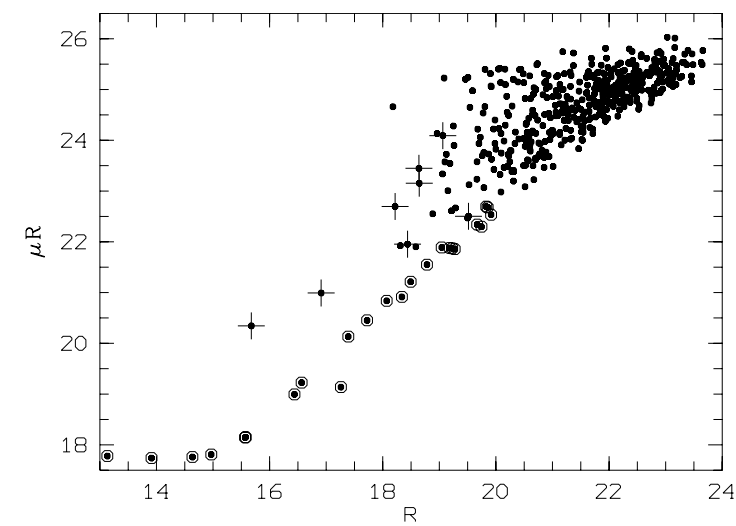

Fig. 4. $R$ central surface brightness versus total $R$ magnitude for the RX J1119 field of view. Objects classified as stars are circled dots. We can see the saturation of the brightest stars. Crossed dots are objects classified as galaxies using spectroscopy.

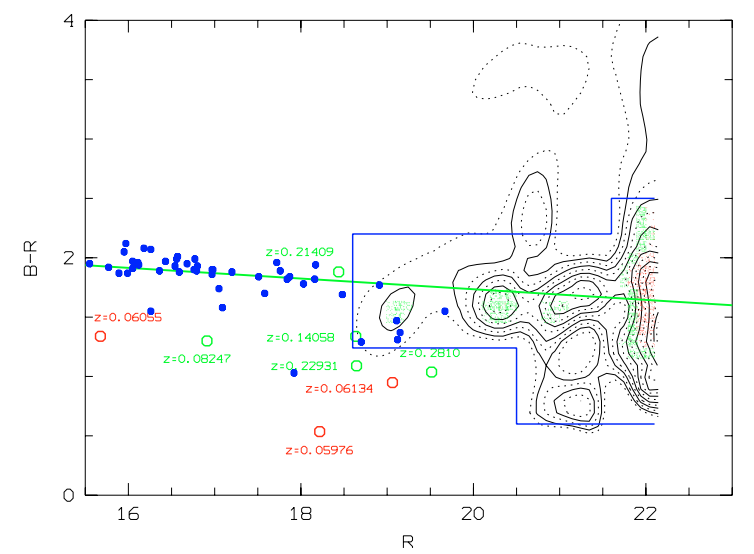

Fig. 5. Total $R$ magnitude versus $B-R$ color for galaxies statistically inside the RX J1119 structure. Galaxies with a known redshift are circles (red: inside the structure, green: outside). Using an adaptative kernel technique, we overplotted the density contours in the $R / B-R$ space. The green line has been normalized to fit the RX J1552 and RX J1416 galaxies in the $B-R / R$ space and to have the Coma cluster slope. Blue dots are galaxies with spectroscopy from the RX J1552 and RX J1416 fossil groups (Mendes de Oliveira et al. 2006, Cypriano et al. 2006) with their magnitudes and colors translated into our system. The bluedelimited area corresponds to our modeling of the positive loci of the density contours. We limit the graph at $R \sim 22$ to take into account the limited completeness of our catalogs.

galaxies per $\mathrm{deg}^{2}$ along the $\mathrm{C} 1, \mathrm{C} 2$ and $\mathrm{C} 2$ lines of sight $\left(N_{\mathrm{C} 123 \text { 1.o. }}\right)$ as:

$N_{\mathrm{RX} J 119}(R, B-R)=N_{\mathrm{RX} J 1119 \text { l.o.s }}-N_{\mathrm{C} 123 \text { l.o.s }}$.

This assumed that the $\mathrm{C} 1, \mathrm{C} 2$ and $\mathrm{C} 3$ fields were representative of the global galaxy population. In order to estimate the uncertainty on the previous difference, we computed the standard deviation $\sigma(R, B-R$ ) of the counts (for given $R$ and $B-R$ ) between the three fields. This gave us an $R / B-R$ map of the uncertainty.

Finally, we divided the $N_{\mathrm{RX} \mathrm{J} 1119}(R, B-R)$ map by the $\sigma(R, B-R)$ map to generate a significance map.

The resulting CMR is shown in Fig. 5. We only plotted contours of the areas where $N_{\mathrm{RX}}$ J1119 1.o.s was greater than $N_{\mathrm{C} 123 \text { l.o.s, }}$, i.e., the places where galaxies are statistically present inside the RX J1119 structure.

First, this figure shows that RX J1119 is indeed a fossil group (besides the fact that its X-ray luminosity estimated by Jones et al. (2003) falls within the allowed range for such groups): the magnitude difference between the brightest and second brightest galaxies in the structure is greater than two magnitudes. The magnitude difference between the brightest galaxy and the bright galaxy peak (at $R \sim 19$ and $B-R \sim 1.6$ ) in Fig. 4 is also greater than two magnitudes. The only galaxy with a magnitude difference less than two is at a redshift of $\sim 0.0825$, and does not belong to the structure.

Second, the presence of a Red Sequence (RS hereafter) in the CMR is a well-known characteristic of massive galaxy systems (see e.g. Godwin \& Peach 1977 or Mazure et al. 1988). The usual negative slope of this RS is a metallicity effect (Kodama \& Arimoto 1997) originating from the higher ability of massive early type galaxies (as opposed to low mass objects) to keep metals against dissipative processes as supernovae, and then to form redder stars. However, only little is known on a possible RS in fossil groups and especially in RX J1119. Figure 5 shows concentrations of galaxies that are similar to a RS. The green-shaded areas are ones where the RX J1119 structure counts are significant between the $2 \sigma$ and $3 \sigma$ levels. The red-shaded areas are significant above the $3 \sigma$ level.

In order to compare with other fossil groups, we superposed on this figure the CMR of RX J1552.2+2013 ( $z=$ $0.136, \mathrm{RX} \mathrm{J} 1552$ hereafter) and RX J1416.4+2315 $(z=0.137$, RX J1416 hereafter, Mendes de Oliveira et al. 2006 and Cypriano et al. 2006). We used Fukugita et al. (1995) to take into account the redshift and filter differences and translated their $A B$ magnitudes into the Vega system. The green line shown in Fig. 5 has been chosen to fit the RX J1552 and RX J1416 galaxies in the $B-R / R$ space and to have a slope of -0.045 (see Adami et al. 2007). The RX J1119 overall RS is in good agreement with those of RX J1552 and RX J1416. A remarkable characteristic of RX J1119 compared to RX J1552 and RX J1416 is that the population of galaxies is very poor. Translated to $z=0.061$, the RX J1416 and RX J1552 fields show a significant population of galaxies between $R=16$ and $R=19$ while the first significant RX J1119 galaxy concentrations occur for $R$ fainter than 18.5 . This is probably related to the fact that RX J1416 and RX J1552 are much more massive than RX J1119 (from the X-ray luminosities given by Jones et al. 2003) and probably underwent a larger field galaxy infall, which compensated more efficiently the galaxy structure depopulation by merging or disruptions. We also note that the brightest galaxy of RX J1119 is bluer than the mean RS, as already observed in RX J1552 and RX J1416 (Mendes de Oliveira et al. 2006; Cypriano et al. 2006).

We can now use the RS in the RX J1119 CMR to select galaxies possibly in RX J1119 and to compute the galaxy density map of the structure. This is done by selecting galaxies inside the blue-delimited region (arbitrarily defined to encompass the positive areas) in Fig. 5. This does not totally prevent us from selecting background galaxies with the same color/magnitude combination, but the contrast between structure and field galaxies is increased roughly threefold. We computed such a galaxy density map in Fig. 6. This figure shows that there is a galaxy concentration close to the X-ray position of RX J1119 (but not centered on it) and with an extension larger than the X-ray halo (see Jones et al. 2003). In order to encourage a possible spectroscopic survey of these faint galaxies, we list them in Table 3.

This concentration is isolated from several other concentrations of galaxies sharing the same colors. Assuming that these galaxies are part of the RX J1119 structure, it therefore seems that the inner core of RX J1119 is also isolated in terms of faint galaxies. The closer external galaxy concentrations are located at a mean distance of $\sim 150 \mathrm{kpc}$ from the central structure. 


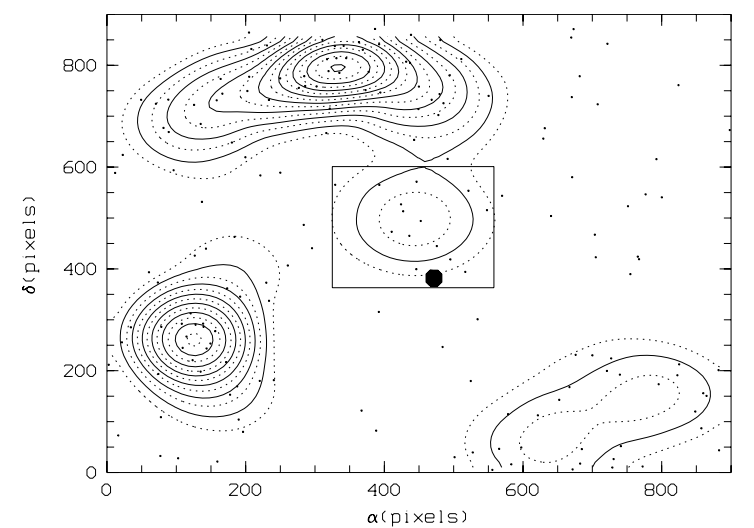

Fig. 6. Adaptative kernel galaxy density map (in pixels) for galaxies inside the blue-delimited area in Fig. 5. The X-ray position of RX J1119 is shown by the filled circle.

Using a bidimensional Kolmogorov-Smirnov test, we show that the galaxies inside and outside the central galaxy concentration of Fig. 6 (and inside the blue-delimited area of Fig. 5) do not have a different distribution inside the $R / B-R$ space (at the $99 \%$ level). Therefore they probably followed similar evolutionary paths. The galaxies very close to the RX J1119 center and at the edges of the structure can therefore be considered as similar.

\section{Conclusions}

Let us summarize our main findings:

- RX J1119 is located at the center of a low-galaxy-density bubble.

- RX J1119 is a fossil group which shows a RS in its CMR, quite similar to RSs of other fossil groups.

- RX J1119 is a very poor and low-mass structure (even compared to other fossil groups) with the first galaxies statistically inside the structure fainter than $R=18.5$.

- The faint RX J1119 galaxy population is strongly clustered and isolated from surrounding galaxy layers. RX J1119 is therefore isolated in terms of bright and faint galaxies, even at small scales. This empty ring seen in Fig. 6 has a radius of 150 to $200 \mathrm{kpc}$. This could be interpreted as the radius where galaxies start to be significantly influenced by the potential well of the fossil group. However, using the formulae relating the virial radius to the X-ray temperature $T$ of the structure (Jones et al. 2003) gives a very low estimate of $T$ (only a fraction of a $\mathrm{keV}$ ). An estimate of $T$ based on X-ray data is therefore needed to confirm or invalidate this interpretation.

Overall, these conclusions are in good agreement with the following building scenario for fossil groups: an isolated galaxy
Table 3. Coordinates, $R$ band magnitude and $B-R$ colors of objects included in the central concentration around RX J1119. They were previously uncataloged in NED and there is no redshift available for these objects.

\begin{tabular}{lll}
\hline \hline Coordinates & $R$ & $B-R$ \\
\hline $11: 19: 50.8,+21: 29: 03.0$ & 21.84 & 1.33 \\
$11: 19: 41.1,+21: 28: 56.0$ & 21.66 & 1.33 \\
$11: 19: 46.0,+21: 28: 36.6$ & 21.67 & 1.92 \\
$11: 19: 41.2,+21: 27: 03.9$ & 21.02 & 1.10 \\
$11: 19: 45.3,+21: 27: 53.2$ & 20.91 & 1.96 \\
$11: 19: 46.6,+21: 27: 58.6$ & 21.20 & 1.73 \\
$11: 19: 44.9,+21: 29: 08.0$ & 21.21 & 1.28 \\
$11: 19: 45.8,+21: 28: 27.0$ & 21.14 & 1.90 \\
$11: 19: 44.8,+21: 27: 07.2$ & 19.08 & 1.67 \\
$11: 19: 42.3,+21: 27: 21.0$ & 19.25 & 1.41 \\
$11: 19: 47.6,+21: 29: 03.2$ & 19.96 & 1.78 \\
$11: 19: 43.3,+21: 27: 39.3$ & 20.21 & 2.19 \\
$11: 19: 39.7,+21: 28: 29.3$ & 20.03 & 1.67 \\
$11: 19: 44.5,+21: 28: 13.8$ & 20.11 & 1.91 \\
\hline
\end{tabular}

structure passively evolving with only minor field contribution due to its location in a low-density environment (see also D'Onghia et al. 2005 and references therein). We now need to put this result on firmer grounds by analysing in a similar way the neighborhood of other known fossil groups.
Acknowledgements. The authors thank the referee for very useful and constructive comments. The authors gratefully acknowledge the contributions of the OHP team and the students of the 2005/2006 class of the Aix-Marseille I Rayonnement, Plasmas et Astrophysique M2.

\section{References}

Adami, C., Picat, J. P., Savine, C., et al. 2006, A\&A, 451, 1159

Adami, C., Durret, F., Mazure, A., et al. 2007, A\&A, 462, 411

Bertin, E., \& Arnouts, S. 1996, A\&AS, 117, 393

Cypriano, E. S., Mendes de Oliveira, C. L., \& Sodré Jr. L. 2006, AJ, 132, 514

D’Onghia, E., Sommer-Larsen, J., Romeo, A. D., et al. 2005, ApJ, 630, L109

Fukugita, M., Shimasaku, K., \& Ichikawa, T. 1995, PASP, 107, 945

Gazelle, F., Robin, A., \& Goidet-Devel, B. 1995, Vist. Astron., 39, 105

Godwin, J. G., \& Peach, J. V. 1977, MNRAS, 181, 323

Hoyle, F., \& Vogeley, M. S. 2004, ApJ, 607, 751

Ilbert, O., Tresse, L., Zucca, E., et al. 2005, A\&A, 439, 863

Jones, L. R., Ponman, T. J., Horton, A., et al. 2003, MNRAS, 343, 627

Kodama, T., \& Arimoto, N. 1997, A\&A, 320, 41

Landolt, A. U. 1992, AJ, 104, 340

Lanzoni, B., Guiderdoni, B., Mamon, G. A., et al. 2005, MNRAS, 361, 369

Mazure, A., Proust, D., Mathez, G., \& Mellier, Y. 1988, A\&AS, 76, 339

Mendes de Oliveira, C., Cypriano, E. S., \& Sodré Jr., L. 2006, AJ, 131, 158

Popesso, P., Bohringer, H., Romaniello, M., \& Voges, W. 2005, A\&A, 433, 415

Sarazin, C. L. 1986, Rev. Mod. Phys., 58, 1

Schlegel, D. J., Finkbeiner, D. P., \& Davis, M. 1998, ApJ, 500, 525

Ulmer, M. P., Adami, C., Covone, G., et al. 2005, ApJ, 624, 124 